\title{
SYBR Green-based Real-Time PCR targeting kinetoplast DNA can be used to discriminate between the main etiologic agents of Brazilian cutaneous and visceral leishmaniases
}

Daniela Pita-Pereira', Rachel Lins², Marcia P Oliveira³, Rosimar B Lima³, Bernardo AS Pereira', Otacilio C Moreira', Reginaldo $\mathrm{PBrazi}^{4}$ and Constança Britto ${ }^{1^{*}}$

\begin{abstract}
Background: Leishmaniases control has been hampered by the unavailability of rapid detection methods and the lack of suitable therapeutic and prophylactic measures. Accurate diagnosis, which can distinguish between Leishmania isolates, is essential for conducting appropriate prognosis, therapy and epidemiology. Molecular methods are currently being employed to detect Leishmania infection and categorize the parasites up to genus, complex or species level. Real-time PCR offers several advantages over traditional PCR, including faster processing time, higher sensitivity and decreased contamination risk.
\end{abstract}

Results: A SYBR Green real-time PCR targeting the conserved region of kinetoplast DNA minicircles was able to differentiate between Leishmania subgenera. A panel of reference strains representing subgenera Leishmania and Viannia was evaluated by the derivative dissociation curve analyses of the amplified fragment. Distinct values for the average melting temperature were observed, being $78.95^{\circ} \mathrm{C} \pm 0.01$ and $77.36^{\circ} \mathrm{C} \pm 0.02$ for Leishmania and Viannia, respectively $(p<0.05)$. Using the Neighbor-Joining method and Kimura 2-parameters, the alignment of 12 sequences from the amplified conserved minicircles segment grouped together L. (V.) braziliensis and L. (V.) shawii with a bootstrap value of 100\%; while for $L$. (L.) infantum and L. (L.) amazonensis, two groups were formed with bootstrap values of $100 \%$ and $62 \%$, respectively. The lower dissociation temperature observed for the subgenus Viannia amplicons could be due to a lower proportion of guanine/cytosine sites (43.6\%) when compared to species from subgenus Leishmania (average of 48.4\%). The method was validated with 30 clinical specimens from visceral or cutaneous leishmaniases patients living in Brazil and also with DNA samples from naturally infected Lutzomyia spp. captured in two Brazilian localities.

Conclusions: For all tested samples, a characteristic amplicon melting profile was evidenced for each Leishmania subgenus, corroborating the data from reference strains. Therefore, the analysis of thermal dissociation curves targeting the conserved kinetoplast DNA minicircles region is able to provide a rapid and reliable method to identify the main etiologic agents of cutaneous and visceral leishmaniases in endemic regions of Brazil.

Keywords: SYBR Green Real-time PCR, Leishmaniases, kinetoplast DNA, thermal dissociation curves, molecular diagnosis, Brazil

\footnotetext{
* Correspondence: cbritto@ioc.fiocruz.br

'Laboratório de Biologia Molecular e Doenças Endêmicas, Instituto Oswaldo

Cruz, FIOCRUZ, Pavilhão Leônidas Deane, sala 209, Avenida Brasil 4365,

Manguinhos, 21040-360 Rio de Janeiro, RJ, Brazil

Full list of author information is available at the end of the article
} 


\section{Background}

The leishmaniases represent a group of diseases with worldwide distribution and a wide spectrum of clinical presentations, which constitute an important public health problem. Their control has been hampered by the unavailability of rapid means of detection and the lack of suitable therapeutic or prophylactic measures. In the Americas, members from the subgenus (Viannia), including Leishmania (Viannia) braziliensis and Leishmania (Viannia) panamensis, and from the Leishmania (Leishmania) mexicana complex cause the majority of cutaneous disease cases, whereas Leishmania (Leishmania) infantum (Syn. L. chagasi) is associated with visceral disease [1,2]. Cutaneous leishmaniasis (CL) in Brazil is caused by a variety of dermotropic Leishmania species and a great diversity of these parasites is found in the Amazon Region. Except in primary forest areas in North Brazil and the Amazon Region, $L .(V$.) braziliensis is the main widespread etiologic agent of CL in Brazil [3].

An accurate diagnostic method that allows the distinction between Leishmania isolates with overlapping geographic distribution is necessary to enable appropriate prognosis, epidemiology and therapy conducts. To this aim, molecular methods have been increasingly employed in an effort to detect infection and categorize Leishmania parasites up to genus, complex or species level [4]. Epidemiological data (clinical presentation and area of endemicity etc) are therefore of prime interest to complement molecular diagnosis. Different PCR-based methods targeting microsatellites, kinetoplastic DNA (kDNA), telomeric sequences, or gp63, hsp70, miniexon, $\beta$-tubulin, and rRNA genes have already been proposed [5]. More recently, real-time PCR, a platform that can process a sample in less than an hour, has been reported to rapidly differentiate even single nucleotide mutations within a target DNA sequence [6-13]. These assays are able to distinguish between groups of Leishmania, such as parasites from the $L$. (L.) donovani complex, and from the subgenus (Viannia) and other species from the subgenus (Leishmania) [10,12]. With the use of TaqMan probes directed to the Leishmania glucose-6-phosphate dehydrogenase locus, it was possible to differentiate $L$. $(V$.$) braziliensis from other Viannia species and from$ those of subgenus Leishmania [7].

The aim of the present study was to evaluate the potential use of the conserved motif of kDNA minicircles in a SYBR Green-based real-time PCR approach, to discriminate between Leishmania subgenera and, therefore identify the main etiologic agents of cutaneous (CL) and visceral (VL) leishmaniases in Brazil, by exploiting differences in the kinetic dissociation profiles of the amplified DNA. The method was successfully applied to identify Leishmania species in 30 clinical samples collected from Brazilian patients with confirmed diagnosis of cutaneous or visceral disease, and also to confirm the infection by $L$. $(V$.) braziliensis or $L$. (L.) infantum in wild sand flies captured in different localities of the country.

\section{Methods}

\section{Organisms, cell culture and DNA extraction}

Promastigotes of Leishmania (V.) braziliensis (MHOM/ BR/1975/M2903), L. (V.) shawi (MCEB/BR/1984/ M8408), L. (V.) guyanensis (MHOM/BR/1975/M4147), L. (V.) lainsoni (MHOM/BR/1981/M6426), L. (V.) naiffi (MDAS/BR/1979/M5533), L. (L.) infantum (Syn. L. chagasi) [2] (MHOM/BR/1974/PP75) and L. (L.) amazonensis (MHOM/BR/1977/LTB0016) were provided by the Leishmania collection from Fiocruz (CLIOC), Rio de Janeiro. Epimastigotes from Trypanosoma cruzi $(\mathrm{Cl}-$ Brener and DM28c strains), Endotrypanum monterogei, Herpetomonas muscarum muscarum (ATCC 30260), Phytomonas sp. and Crithidia fasciculata (ATCC11745), were obtained from the Protozoa collection from Fiocruz (COLPROT), Rio de Janeiro. Parasites were cultivated in $3.7 \%$ brain heart infusion (BHI) medium supplemented with $10 \%$ heat-inactivated fetal bovine serum, penicillin $500 \mathrm{U} / \mathrm{mL}$ and streptomycin $500 \mathrm{mg} /$ $\mathrm{mL}$ at $28^{\circ} \mathrm{C}$ for 4 days to reach late-log phase growth. Cells were harvested, washed and resuspended in phosphate-buffered saline (PBS) pH 7.4. Cell pellets containing $300 \mu \mathrm{L}$ PBS were incubated with proteinase $\mathrm{K}$ (at a final concentration of $300 \mu \mathrm{g} / \mathrm{mL}$ ) at $55^{\circ} \mathrm{C}$ for $2 \mathrm{~h}$, and DNA was extracted by the sodium dodecyl sulfate/phenol extraction method [14]. The extracted DNA was precipitated with ethanol, dissolved in $50 \mu \mathrm{L}$ of TE buffer (10 mM Tris-HCl, $1 \mathrm{mM}$ EDTA) and quantified by measuring absorbance at $260 \mathrm{~nm}$.

\section{Clinical specimens}

$5 \mathrm{mM}$ EDTA-supplemented peripheral blood and bone marrow aspirates, as well as tissue biopsy specimens stored in $0.9 \%$ sodium chloride solution, were obtained from 30 patients with confirmed leishmaniasis, as follows. Fifteen of these patients were children considered to be VL carriers, living in the municipality of Campo Grande (Mato Grosso do Sul State) with ages ranging from 2-13 years old, and presenting characteristic clinical features (fever, hepatosplenomegaly and pancytopenia) associated to the detection of parasites in bone marrow aspirates by means of direct microscopy or in NNN (Novy-MacNeal-Nicolle) culture, and/or positive serology $(\geq 1: 80)$ as determined by indirect immunofluorescence (IIF). The remaining fifteen patients were from the municipality of Rio de Janeiro (Rio de Janeiro State), inhabiting areas with well-known occurrences of $C L$ related to $L$. (V.) braziliensis and previously diagnosed by Giemsa staining of biopsy smears, 
histopathological examination of biopsies, or culture and/or PCR-RFLP on DNA extracted from lesion biopsies [15]. Samples corresponding to $1 \mathrm{~mm}^{3}$ of skin biopsies obtained from the borders of the lesions, as well as $300 \mu \mathrm{L}$ of EDTA-supplemented peripheral blood or bone marrow aspirates were used for DNA isolation, according to previously described protocols $[15,16]$. All DNA samples from clinical specimens were kindly provided by the National Reference Laboratory in Molecular Diagnosis of Leishmaniases, Instituto Oswaldo Cruz/ Fiocruz, Rio de Janeiro (approved by the Ethics Committee of Fiocruz - protocol No. 503/09).

\section{Lutzomyia sand fly samples}

Wild sand flies positive for infection with either $L .(V$. braziliensis or $L$. (L.) infantum were divided into pools (10 insects per pool). DNA extraction and multiplex PCR assays followed by isotopic hybridization with specific Leishmania probes were performed as previously described $[17,18]$. A total of eleven positive Lutzomyia female pools were evaluated, consisting of: (i) five $L u$. intermedia pools and three Lu. migonei pools collected in 2003, in two areas from the municipality of Rio de Janeiro (Rio de Janeiro State) with notification of CL [17]; (ii) two Lu. cruzi and one Lu. forattinii pools collected in an endemic area of VL in the municipality of Corumbá (Mato Grosso do Sul State), in May/June 2006 [18].

\section{Real-time PCR assays}

SYBR Green-based real-time PCR was performed with primers directed to the conserved motif of Leishmania kinetoplast DNA minicircles [5'-GGC CCA CTA TAT TAC ACC AAC CCC-3' and 5'-GGG GTA GGG GCG TTC TGC GAA-3'] [19]. The reaction mixture contained 1× Power SYBR Green Master Mix (Applied Biosystems, Foster City, CA, USA), 1 pmol of each primer, $25 \mathrm{ng}$ of template DNA and distilled ultra-pure water for a final reaction volume of $15 \mu \mathrm{L}$. The reactions were set up, in triplicate, in a 96-well optical reaction plate in an ABI Prism 7500 Sequence Detection System (Applied Biosystems, Foster City, CA, USA). The PCR conditions were as follows: an initial 12-min incubation step at $94^{\circ}$ $\mathrm{C}$, followed by 35 cycles of $30 \mathrm{~s}$ at $94^{\circ} \mathrm{C}, 30 \mathrm{~s}$ at $55^{\circ} \mathrm{C}$, and $30 \mathrm{~s}$ at $72^{\circ} \mathrm{C}$. The generation of amplification plots and dissociation kinetic analyses were according to the manufacturer's protocol. After amplification, the melting curve test was performed with an initial denaturation step at $95^{\circ} \mathrm{C}$ for $5 \mathrm{~s}$, followed by 15 seconds at $50^{\circ} \mathrm{C}$ and continuous heating at $0.1^{\circ} \mathrm{C} / \mathrm{s}$ to $90^{\circ} \mathrm{C}$. Calculation of the melting temperature for each amplicon $(T m)$ was done directly by the equipment software. Each assay was repeated at least 3 times to check the reproducibility and reliability. Stringent measures to control sample contamination included two non template negative controls (NTC - reaction mix without DNA and distilled water alone) and DNA obtained from healthy donors' peripheral blood or from uninfected Lutzomyia (male specimen), in each reaction plate. Positive controls (50 ng Leishmania DNA) were also included.

\section{Conventional PCR for cloning}

DNA samples from four Leishmania reference strains $L$. (V.) braziliensis, $L$. (V.) shawi, $L$. (L.) infantum and $L$. $(L$.$) amazonensis, were submitted to a conventional PCR$ using the same primer pair described for the real-time PCR. The $50 \mu \mathrm{L}$ reaction consisted of $5 \mu \mathrm{L}$ DNA template, 5 pmol primers, $1 \times$ Taq polymerase buffer, $1.25 \mathrm{U}$ Taq DNA polymerase (Applied Biosystems, Foster City, CA, USA), $4.5 \mathrm{mM} \mathrm{MgCl} 2,100 \mu \mathrm{M}$ of each dNTP (Invitrogen Corporation, Carlsbad, CA, USA) and distilled ultra-pure water. The mixture was incubated in a Perkin-Elmer thermocycler (GeneAmp PCR System 9600; Applied Biosystems, Foster City, CA, USA), following the same amplification conditions reported for the realtime PCR. Ten microlitres of amplification product were resolved in 2\% agarose gel electrophoresis, stained with GelRed $^{\circledR}$ (Sigma-Aldrich, St. Louis, MO, USA) and visualized under UV-light. The remaining $40 \mu \mathrm{L}$ were purified using the commercial kit Wizard ${ }^{\circledR}$ SV Gel and PCR Clean-up System (Promega, Madison, WI, USA), according to the manufacturer's protocol. After DNA quantification by spectrophotometry, the fragments were cloned using TOPO TA cloning kit ${ }^{\circledR}$ (Invitrogen Corporation, Carlsbad, CA, USA) following the manufacturer's recommendation.

\section{Sequence Analysis}

For each Leishmania reference strain, three clones derived from the amplified conserved minicircles region were sequenced using the BigDye Terminator Cycle Sequencing Kit Ready Reaction version 3.1 on the ABI Prism 3100 Avant Genetic Analyzer (Perkin-Elmer, Applied Biosystems, Foster City, CA, USA), following the manufacturer's specifications. Sequence homology searches were made by the online NCBI BLAST software http://blast.ncbi.nlm.nih.gov/Blast.cgi. Sequences were aligned using the CLUSTAL software in the BioEdit software package [20]. A tree was constructed using the MEGA 3.1 software [21], applying the NeighborJoining method and Kimura 2-parameters. Stability of the tree was confirmed by bootstrap analysis.

\section{Results and discussion}

Limited nucleotide variation of the KDNA conserved motifs promote distinctive melting profiles for Leishmania subgenera

Genomic DNA extracted from four Leishmania promastigote reference strains, representing both Viannia and 
Leishmania subgenera, was assayed. For each subgenus, the SYBR Green melting curve analysis showed a single peak indicating its specificity, which was confirmed by fractionation in an agarose gel. Figure 1 demonstrates the typical dissociation curves representing specific melting temperatures ( $\mathrm{Tm}$ ) generated by the kDNA amplified products of promastigote reference strains from Viannia and Leishmania subgenera [Figure 1- A1 and A2, respectively]. Leishmania (L.) infantum and $L$. (L.) amazonensis, both from subgenus Leishmania, presented an average $\mathrm{Tm}$ of $78.95^{\circ} \mathrm{C} \pm 0.01$, and the two Viannia species, L. (V.) braziliensis and $L$. (V.) shawi, revealed a slightly lower but statistically distinct $\mathrm{Tm}$ value of $77.34^{\circ} \mathrm{C} \pm 0.01$ ( $\mathrm{p}<0.001$ - Mann-Whitney Rank Sum Test).

Considering an estimated length for the Leishmania minicircles conserved region of around 120 base pairs, we further investigated these minor differences on the melting temperature for each subgenus by sequencing the amplification products obtained from the analyzed Leishmania species. A kDNA-based conventional PCR was performed and the amplified 120 bp fragments were cloned. Three clones were selected for sequencing for each Leishmania species and 12 generated sequences were aligned, revealing slight variations (nucleotide substitutions, insertions and/or deletions). All 12 sequences are shown in an additional file [see Additional file 1]. Despite the limited number of nucleotide variations in the Leishmania kDNA conserved motif, it was possible to construct a tree based on the Neighbor-Joining method and Kimura 2-parameters [Figure 2]. We observed the formation of a very distinct group with a bootstrap value of $100 \%$ comprising $L$. (V.) shawi and $L$. $(V$.) braziliensis, from the subgenus Viannia. This group revealed in its sequence composition a lower G/C content $(43.6 \%)$ when compared to reference strains from

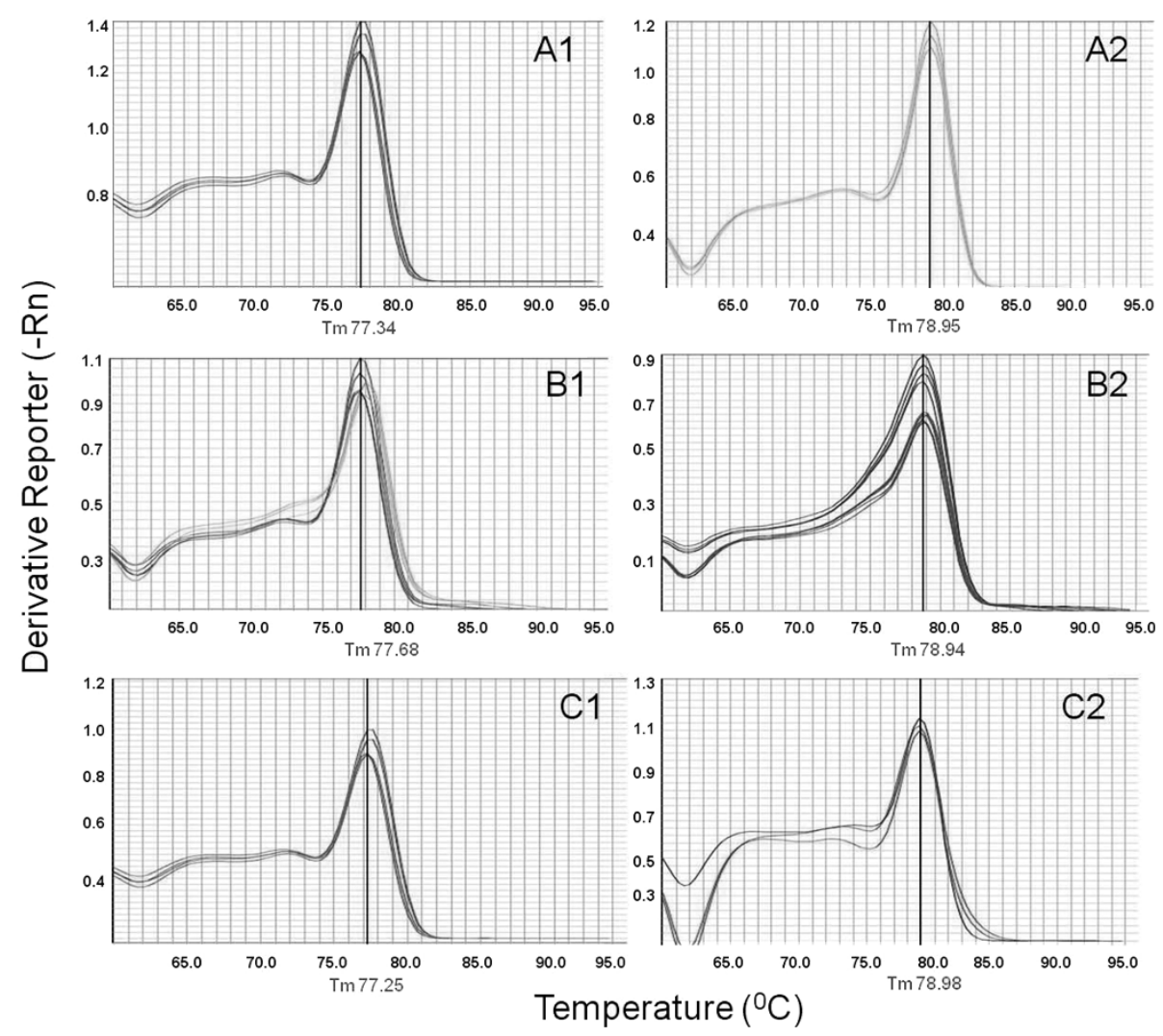

Figure 1 Melting curve analyses of kDNA conserved regions amplicons. Representative SYBR Green dissociation curves of kDNA amplified products to determinate the melting temperature values [Tm]. Following amplification in the ABI Prism 7500 Sequence Detection System, the amplicons were submitted to a gradual temperature increase $\left(0.1^{\circ} \mathrm{C} / \mathrm{s}\right.$ to $\left.90^{\circ} \mathrm{C}\right)$. The $\mathrm{Tm}$ calculation of each amplicon was done directly by the software provided (indicated by the perpendicular line in the graphics). Each trace in the charts represents a single analyzed DNA sample. The graphics on the left represent typical kinetic dissociation profiles obtained for the Viannia subgenus: (A1) L. (V.) shawi and L. (V.) braziliensis promastigote reference strains; (B1) clinical samples from cutaneous disease patients; (C1) Lu. intermedia and Lu. migonei pools naturally infected by $L$. (V.) braziliensis. On the right are represented the melting profiles for the subgenus Leishmania: (A2) L. (L.) amazonensis and $L$. (L.) infantum promastigote reference strains; (B2) clinical specimens from visceral disease patients; (C2) Lu. cruzi and Lu. forattinii pools naturally infected by $L$. (L.) infantum. 


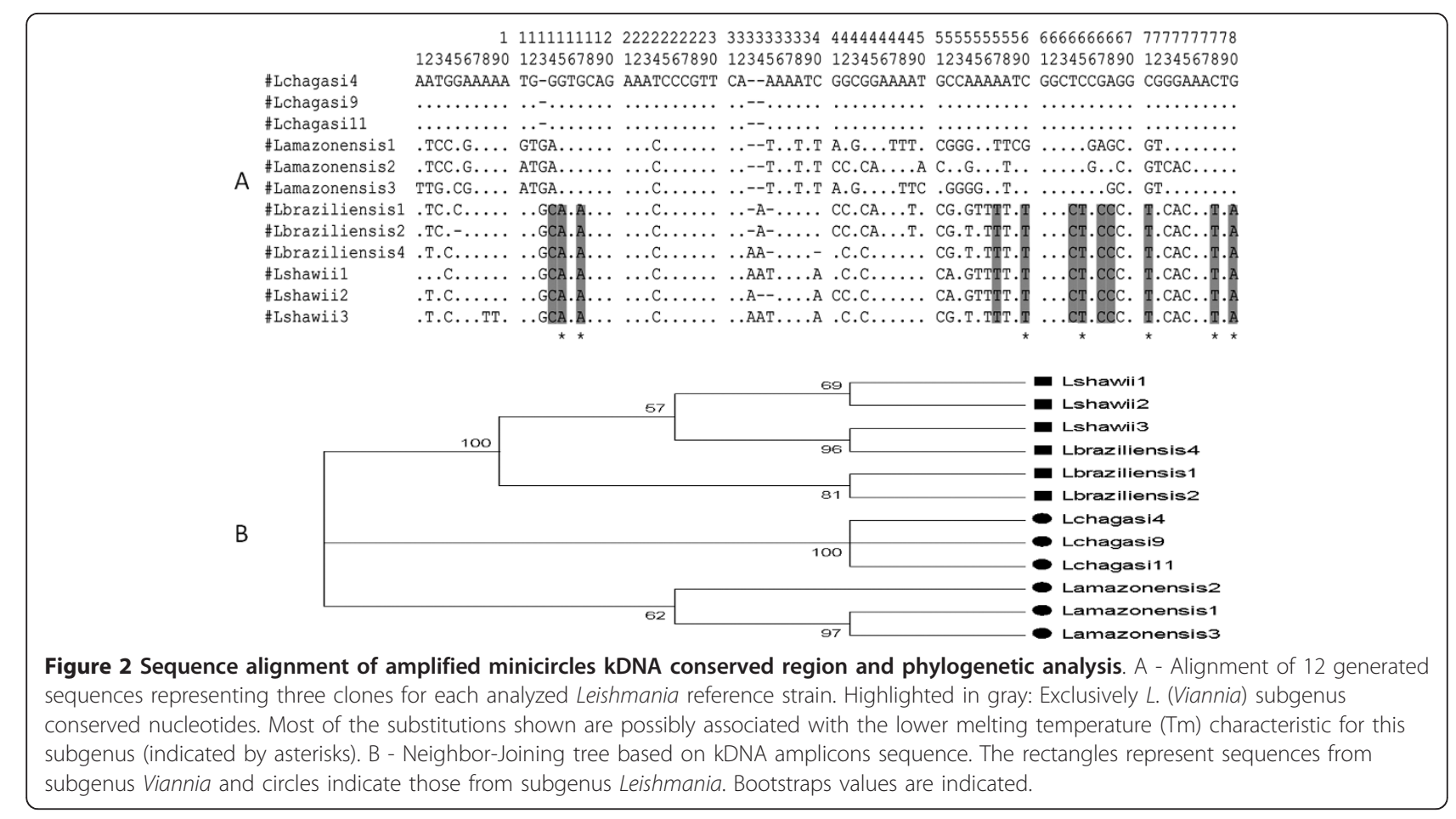

the subgenus Leishmania: L. (L.) infantum (48.1\%) and L. (L.) amazonensis (48.7\%). Taking into consideration that the kDNA amplified fragments were of similar lengths, this difference in G/C ratio could explain the lower dissociation temperature observed for DNA amplicons derived from subgenus Viannia $\left(77.34^{\circ} \mathrm{C} \pm 0.01\right)$. For the Leishmania subgenus, both analyzed species formed groups with significant bootstrap values $-100 \%$ for $L$. (L.) infantum and $62 \%$ for L. (L.) amazonensis [Figure 2]. Differences in base composition between these two species did not interfere with the dissociation curve kinetics calculated for the respective amplified products.

In order to verify if other Viannia species causing CL in Brazil generated the characteristic melting profiles for the kDNA amplicons previously observed for this subgenus, the panel of Viannia reference strains was expanded by including $L$. (V.) guyanensis, $L$. (V.) naiffi and $L$. (V.) lainsoni promastigotes [see Additional file 2]. No significant differences were observed between the Tm values for these species, with an average of $77.36^{\circ} \mathrm{C}$ \pm 0.02 ( $\mathrm{p}>0.05$ ). Table 1 presents the Tm values generated from the dissociation curve analysis for each tested Leishmania reference strain from both subgenera. Even though different species from the same subgenus presented similar melting points, the melting curve analyses were able to distinguish between the two main etiologic agents of cutaneous and visceral leishmaniases in Brazil, $L$. (V.) braziliensis and L. (L.) infantum, respectively.

\section{Methodology validation with human clinical specimens} and wild sand flies

The findings revealed through the analyses of Leishmania promastigote reference strains were corroborated with the inclusion of DNA samples obtained from clinical specimens (peripheral blood, bone marrow aspirates and skin biopsies) of patients living in Brazilian leishmaniasis endemic areas and with confirmed diagnosis of visceral or cutaneous disease. To ensure the reproducibility of the assays, positive controls - DNA from $L$. $(V$.) braziliensis, L. (V.) shawi, L. (L.) infantum and $L$. (L.) amazonensis reference strains, were included in each run. Figure 1 shows the characteristic kinetic dissociation profiles for the kDNA amplicons derived from human clinical samples. The results with the VL samples indicated a typical Tm value for the subgenus Leishmania $\left(78.94^{\circ} \mathrm{C} \pm 0.37\right)$ [Figure $1, \mathrm{~B} 2$ ], which is differentiated from the Tm observed for the analyzed dermotropic clinical specimens $\left(77.68^{\circ} \mathrm{C} \pm 0.38\right)$ [Figure 1 , $\mathrm{B} 1]$. These data suggest that the technique of dissociation curve analysis has the potential to be applied to discriminate between $L$. (V.) braziliensis and $L$. (L.) amazonensis infection in areas of Brazil where the distribution of these species overlaps. As for cases of visceral disease in Brazilian patients, this methodology is able to identify the etiologic agent $L$. (L.) infantum. The specificity of the method was confirmed with DNA extracted from healthy donors peripheral blood. 
Table 1 Differentiation between Leishmania subgenera by SYBR Green melting temperatures (Tm) from amplified minicircles kDNA conserved region

\begin{tabular}{|c|c|c|c|}
\hline Source & Samples & Origin & $\begin{array}{l}\text { Melting Temperature } \\
\left({ }^{\circ} \mathrm{C} \pm \mathrm{SD}\right)\end{array}$ \\
\hline \multirow[t]{7}{*}{ Promastigote reference strains } & L (V.) braziliensis (MHOM/BR/1975/M2903) & IOC/FIOCRUZ & $77.34 \pm 0.01$ \\
\hline & L (V.) shawi (MCEB/BR/1984/M8408) & IOC/FIOCRUZ & $77.34 \pm 0.01$ \\
\hline & L (V.) naiffi (MDAS/BR/1979/M5533) & IOC/FIOCRUZ & $77.34 \pm 0.01$ \\
\hline & L (V.) lainsoni (MHOM/BR/1981/M6426) & IOC/FIOCRUZ & $77.37 \pm 0.01$ \\
\hline & L(V.) guyanensis (MHOM/BR/1975/M4147) & IOC/FIOCRUZ & $77.39 \pm 0.01$ \\
\hline & L (L.) infantum (Syn. L. chagasi) (MHOM/BR/1974/PP75) & IOC/FIOCRUZ & $78.95 \pm 0.01$ \\
\hline & L (L.) amazonensis (MHOM/BR/1977/LTB0016) & IOC/FIOCRUZ & $78.94 \pm 0.00$ \\
\hline \multirow[t]{2}{*}{ Human clinical specimens } & Cutaneous leishmaniasis (skin biopsies) & Rio de Janeiro/RJ & $77.68 \pm 0.38$ \\
\hline & Visceral leishmaniasis (peripheral blood and bone marrow aspirates) & Campo Grande/MS & $78.94 \pm 0.37$ \\
\hline \multirow[t]{4}{*}{ Field sand flies } & Lutzomyia intermedia & Rio de Janeiro/RJ & $77.25 \pm 0.15$ \\
\hline & Lutzomyia migonei & Rio de Janeiro/RJ & $77.25 \pm 0.15$ \\
\hline & Lutzomyia cruzi & Corumbá/MS & $78.98 \pm 0.10$ \\
\hline & Lutzomyia forattini & Corumbá/MS & $78.98 \pm 0.10$ \\
\hline
\end{tabular}

IOC: Oswaldo Cruz Institute, FIOCRUZ: Oswaldo Cruz Foundation, RJ: State of Rio de Janeiro, MS: State of Mato Grosso do Sul, SD: standard deviation The melting temperatures of kDNA amplified products were analyzed from samples obtained from Leishmania promastigotes reference strains, clinical specimens from cutaneous and visceral leishmaniases patients and field-captured phlebotomine insects. The dissociation curves analyses revealed distinct melting temperatures between the subgenera Viannia and Leishmania $(\mathrm{p}<0.001$ - Mann-Whitney Rank Sum Test).

The molecular method was also able to differentiate between natural infections caused by $L$. (V.) braziliensis or L. (L.) infantum in Lutzomyia sand flies, which were previously positive in a diagnostic assay by multiplex conventional PCR following hybridization $[17,18]$. The assays were performed with DNA samples obtained from five pools of Lu. intermedia and three pools of Lu. migonei (10 insects/pool) collected in the municipality of Rio de Janeiro, in areas with notification of CL in human and dogs [17]; and, also, DNA extracted from two pools of $L u$. cruzi and one pool of Lu. forattinii from specimens captured in an endemic VL area in the municipality of Corumbá, Mato Grosso do Sul State [18]. As displayed in Figure 1 the data from these specimens were in accordance with our findings, revealing kinetic dissociation profiles compatible with the characteristic Tm previously observed for $L$. (V.) braziliensis $\left(77.25^{\circ} \mathrm{C} \pm 0.15\right)$ in pools of $L u$. intermedia/Lu. migonei [Figure 1, C1], and for L. (L.) infantum $\left(78.98^{\circ} \mathrm{C} \pm 0.10\right)$ in pools of $L u$. cruzi/Lu. forattinii [Figure 1, C2].

DNAs from other Trypanosomatids that also parasitize the hosts of Leishmania, such as Trypanosoma cruzi (mammalian host) and Endotrypanum (invertebrate host), as well as other species closely related (Herpetomonas, Phytomonas, Crithidia) were tested for the specificity of the assay. DNA from uninfected Lutzomyia was also assayed as a negative control. There was no amplification of DNA in these assays.

Table 1 summarizes the data obtained through the analyses of cultivated Leishmania promastigotes, human clinical specimens and naturally infected phlebotomine sand flies.

\section{Molecular markers and PCR-based systems for the diagnosis of Leishmania infection}

Restriction fragment length polymorphism (RFLP) analyses of PCR-amplified products from multicopy genes have shown promising results in detecting Leishmania species and in clarifying the molecular diversity and relationships within Leishmania spp. [22-24]. PCR-based methods with further molecular typing by sequence analysis have also been described [25,26]. Real-time PCR is currently considered as an emerging technology for the detection, genetic characterization and quantification of protozoan parasites. By using the Light-Cycler SYBR Green system targeting minicircles kDNA, Nicolas et al. (2002) were able to differentiate four important Leishmania species from the Old World [8]. More recently, the performance of PCR-RFLP for the internal transcribed spacer of ribosomal RNA (ITS1) and SYBR Green-based real-time PCR focused on kDNA were compared, with the aim of identifying $L$. (L.) infantum, L. (L.) major and L. (L.) tropica/killicki, the etiologic agents of CL in Tunis, directly from human skin scrapings [6]. This comparison revealed a failure of the kDNA real-time PCR method in identifying the Tunisian L. (L.) tropica/L. (L.) killicki in 5 out of 27 samples, which the authors attributed to the kinetoplast DNA polymorphism found in Leishmania strains. Thus, it was suggested that standardization of kDNA real-time PCR is needed to allow interlaboratory comparisons and 
maximize repeatability. A recent investigation reported the discriminatory power of a new high-resolution tool for the dissociation analysis of PCR products derived from the ITS1 gene [11]. The high resolution melt analysis (HRM) could distinguish between all Old World Leishmania species causing human disease, except $L$. (L.) infantum from $L$. (L.) donovani, which presented similar HRM curves. Other studies also demonstrated the ability of melting curve analysis to distinguish Leishmania parasites up to subgenus, complex or species level, through the use of different targets, such as the $18 \mathrm{~S}$ rDNA sequences [10], gp63 [27] and glucose-6phosphate dehydrogenase (g6pd) [7]. Alternatively, a TaqMan-based real-time PCR for the detection of glucosephosphate isomerase (GPI) gene was able to discriminate between four Leishmania groups - subgenus Viannia and the complexes L. (L.) mexicana, L. (L.) donovani/infantum and L. (L.) major [12].

Accurate and sensitive procedures for the diagnosis of Leishmania infection and species identification directly from clinical material or through the analysis of phlebotomine sand flies are still required to enable adequate treatment and appropriate leishmaniases control. Identification of the prevalent Leishmania species in sand flies can alert clinicians to potential subsequent human cases in a determined geographic area. In this sense, the use of kDNA minicircles on PCR-based assays coupled to isotopic hybridization has been shown to offer enhanced sensitivity over more traditional diagnostic methods to evaluate natural infection in sand fly vectors from Brazil, allowing DNA detection corresponding to only one Leishmania parasite presented in pools of 10 male phlebotomine insects in reconstituted samples [17]. This high sensitivity is partly due to the elevated copy number of the target kDNA minicircles, which are represented in approximately 10,000 copies per parasite genome. Compared with conventional PCR tests targeting kinetoplast DNA, the most important implication of the assay herein reported is the ability to rapidly discriminate among the pathogenic species representing the most common causative agents associated to $\mathrm{CL}$ or $\mathrm{VL}$ in Brazil, thus optimizing the identification of Leishmania DNA in sand flies or clinical specimens without the need of posterior detection of amplicons by gel electrophoresis or hybridization step.

PCR-based assays directed to kDNA are currently the most sensitive methodology for the diagnosis of either visceral or cutaneous leishmaniasis [28]. The samples tested in the present study were selected, as they were positive by traditional diagnostic methods (microscopy/ culture and/or serology, Giemsa staining/histopathology), as well as PCR, before being subjected to testing with this assay. In all cases, the SYBR Green melting curve profile was able to corroborate the predetermined diagnosis. As formerly reported, the existence of shifted and overlapping $T m$ values indicates kinetoplast DNA polymorphism among Leishmania strains, which causes a variation of the amplified sequence and, consequently, of the corresponding melting temperature [6]. Nevertheless, the kDNA polymorphism observed in the present study is too limited to allow the use of SYBR Greenbased real-time PCR as an intraspecific typing method. Other methods, such as the analysis of microsatellite markers, are more appropriate for this specific issue [29].

\section{Conclusions}

Based on the sequence-dependent thermal dissociation properties, despite the analysis of a relatively small sample set, our data suggest that SYBR Green-based realtime PCR targeting the conserved minicircles region from kinetoplast DNA provides a rapid, sensitive and simple alternative for the precise identification of Leishmania subgenera. This methodology was able to discriminate among the human-pathogenic species representing the most common causative agents of cutaneous and visceral leishmaniases in Brazilian endemic areas. Similar kinetic dissociation profiles were observed for the amplified products derived from subgenus Viannia reference strains, skin biopsies from L. (V.) braziliensis-related CL patients and Lutzomyia sand flies infected by $L$. (V.) braziliensis. Likewise, the analyses performed with promastigotes from subgenus Leishmania were in accordance to the results obtained with the set of clinical specimens from VL patients and sand fly vectors infected by $L$. (L.) infantum.

\section{Additional material}

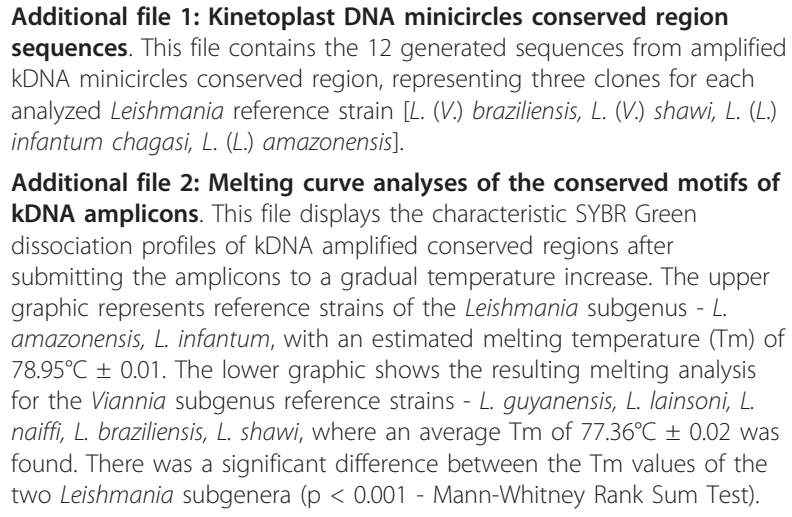
kDNA minicircles conserved region, representing three clones for each analyzed Leishmania reference strain [L. (V.) braziliensis, L. (V.) shawi, L. (L.) infantum chagasi, L. (L.) amazonensis].

Additional file 2: Melting curve analyses of the conserved motifs of kDNA amplicons. This file displays the characteristic SYBR Green dissociation profiles of kDNA amplified conserved regions after submitting the amplicons to a gradual temperature increase. The upper graphic represents reference strains of the Leishmania subgenus - $L$. amazonensis, $L$. infantum, with an estimated melting temperature $(\mathrm{Tm})$ of $78.95^{\circ} \mathrm{C} \pm 0.01$. The lower graphic shows the resulting melting analysis for the Viannia subgenus reference strains - L. guyanensis, L. lainsoni, L. naiffi, L. braziliensis, L. shawi, where an average $T m$ of $77.36^{\circ} \mathrm{C} \pm 0.02$ was found. There was a significant difference between the Tm values of the two Leishmania subgenera ( $p<0.001$ - Mann-Whitney Rank Sum Test).

\section{List of abbreviations}

kDNA: kinetoplast DNA; SYBR Green: N', N'-dimethyl-N-[4-[(E)-(3-methyl-1,3benzothiazol-2-ylidene) methyl]-1-phenylquinolin-1-ium-2-yl]-Npropylpropane-1,3-diamine; PCR: Polymerase Chain Reaction; Tm: melting temperature; CL: Cutaneus Leishmaniasis; VL: Visceral Leishmaniasis; gp63: 
major surface glycoprotein of 63-kDa; hsp70: heat shock protein of 70-kDa; rRNA: ribosomal RNA; G/C: guanine/cytosine; RFLP: Restriction fragment length polymorphism; ITS1: Internal Transcribed Spacer; 185 rDNA: small subunit of the ribosomal DNA; EDTA: Ethylenediamine tetraacetic acid.

\section{Acknowledgements}

The authors are grateful to the Leishmania collection (CLIOC) and the Protozoa collection (COLPROT), both from Instituto Oswaldo Cruz/Fiocruz, Rio de Janeiro, for providing the Leishmania reference strains and closely related trypanosomatids; to Maria Angelica Batista Cardoso for the support on the Real Time PCR-based platform; to the Program for Technological Development in Tools for Health (PDTIS-Fiocruz) for the facilities, and to Dr. Daniel Bray for the kindly English revision. This investigation was supported by the Conselho Nacional de Desenvolvimento Científico e Tecnológico CNPq (grant no. 471797/08-1) and FAPERJ (grant no. E-26/110.257/2010). Daniela Pita-Pereira is a post doctoral fellow of CNPq; Bernardo Pereira is a post doctoral fellow of CAPES/Faperj; Constança Britto receives a fellowship from CNPq.

\section{Author details}

'Laboratório de Biologia Molecular e Doenças Endêmicas, Instituto Oswaldo Cruz, FIOCRUZ, Pavilhão Leônidas Deane, sala 209, Avenida Brasil 4365, Manguinhos, 21040-360 Rio de Janeiro, RJ, Brazil. 'Laboratório de Biologia Molecular de Insetos, Instituto Oswaldo Cruz, FIOCRUZ, Rio de Janeiro, RJ, Brazil. 'Laboratório Interdisciplinar em Pesquisas Médicas, Instituto Oswaldo

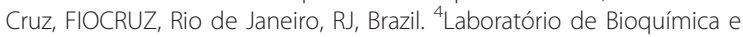
Fisiologia de Insetos, Instituto Oswaldo Cruz, FIOCRUZ, Rio de Janeiro, RJ, Brazil.

\section{Authors' contributions}

DPP designed the experiments, carried out the molecular studies (PCR-based assays and sequencing), participated in the sequence alignment, and helped to draft the manuscript. RL participated with the sequence alignment and with the construction and interpretation of the tree. MPO and RBL provided the DNA samples from human clinical specimens, and were responsible for performing all previous diagnostic tests for the $\mathrm{CL}$ and $\mathrm{VL}$ patients included in this study. BASP and OCM participated with the statistical analysis, adaptation of figures and table, and revising the English grammar. RPB contributed to sand fly captures and the taxonomic identification of Lutzomyia specimens. CB conceived and designed the study, and wrote the final version of the manuscript. All authors read and approved the final manuscript.

\section{Competing interests}

The authors declare that they have no competing interests.

Received: 26 August 2011 Accepted: 12 January 2012 Published: 12 January 2012

\section{References}

1. Cupolillo E, Medina-Acosta E, Noyes H, Momen H, Grimaldi G Jr: A revised classification for Leishmania and Endotrypanum. Parasitol Today 2000, 16:142-144.

2. Kuhls K, Alam MZ, Cupolillo E, Ferreira GEM, Mauricio IL, Oddone R, Feliciangeli MD, Wirth T, Miles MA, Schönian G: Comparative microsatellite typing of New World Leishmania infantum reveals low heterogeneity among populations and its recent Old World origin. PLoS Negl Trop Dis 2011, 5(6):e1155

3. Rangel EF, Lainson R: Proven and putative vectors of American cutaneous leishmaniasis in Brazil: aspects of their biology and vectorial competence. Mem Inst Oswaldo Cruz 2009, 104(suppl 7):937-954.

4. Schönian G, Mauricio I, Gramiccia M, Cañavate C, Boelaert M, Dujardin JC: Leishmaniases in the Mediterranean in the era of molecular epidemiology. Trends Parasitol 2008, 24:135-142.

5. Tavares CA, Fernandes AP, Melo MN: Molecular diagnosis of leishmaniasis. Expert Rev Mol Diagn 2003, 3:657-667.

6. Ben Abda I, Monbrison F, Bousslimia N, Aoun K, Bouratbinea A, Picot S: Advantages and limits of real-time PCR assay and PCR-restriction fragment length polymorphism for the identification of cutaneous Leishmania species in Tunisia. Trans R Soc Trop Med Hyg 2010, 105(suppl 1):17-22.
7. Castilho TM, Camargo LM, McMahon-Pratt D, Shaw JJ, Floeter-Winter LM: A real-time polymerase chain reaction assay for the identification and quantification of American Leishmania species on the basis of glucose-6phosphate dehydrogenase. Am J Trop Med Hyg 2008, 78(suppl 1):122-132.

8. Nicolas L, Milon G, Prina E: Rapid differentiation of Old World Leishmania species by LightCycler polymerase chain reaction and melting curve analysis. J Microbiol Methods 2002, 51(suppl 3):295-299.

9. Quispe-Tintaya KW, Laurent T, Decuypere S, Hide M, Banuls AL, Doncker SD, Rijal S, Canavate C, Campino L, Dujardin JC: Fluorogenic assay for molecular typing of the Leishmania donovani complex: taxonomic and clinical applications. J Infect Dis 2005, 192:685-691.

10. Schulz A, Mellenthin K, Schönian G, Fleischer B, Drosten C: Detection, differentiation, and quantitation of pathogenic leishmania organisms by a fluorescence resonance energy transfer-based real-time PCR assay. J Clin Microbiol 2003, 41(suppl 4):1529-1535.

11. Talmi-Frank D, Nasereddin A, Schnur LF, Schönian G, Töz SO, Jaffe CL, Baneth G: Detection and identification of Old World Leishmania by high resolution melt analysis. PLoS Negl Trop Dis 2010, 4:e581.

12. Wortmann G, Hochberg L, Houng HH, Sweeney C, Zapor M, Aronson N, Weina P, Ockenhouse CF: Rapid identification of Leishmania complexes by a real-time PCR assay. Am J Trop Med Hyg 2005, 73(suppl 6):999-1004.

13. Wortmann G, Hochberg LP, Arana BA, Rizzo NR, Arana F, Ryan JR: Diagnosis of cutaneous leishmaniasis in Guatemala using a real-time polymerase chain reaction assay and the smartcycler. Am J Trop Med Hyg 2007, 76(suppl 5):906-908.

14. Uliana SR, Affonso MH, Camargo EP, Floeter-Winter LM: Leishmania: genus identification based on a specific sequence of the $18 \mathrm{~S}$ ribosomal RNA sequence. Exp Parasitol 1991, 72:157-163.

15. Pirmez C, da Silva Trajano V, Paes-Oliveira Neto M, Da-Cruz AM, Gonçalvesda-Costa SC, Catanho M, Degrave W, Fernandes O: Use of PCR in diagnosis of human american tegumentary leishmaniasis in Rio de Janeiro, Brazil. J Clin Microbiol 1999, 37(suppl 6):1819-1823.

16. Fraga TL, Brustoloni YM, Lima RB, Dorval ME, Oshiro ET, Oliveira J, Oliveira AL, Pirmez C: Polymerase chain reaction of peripheral blood as a tool for the diagnosis of visceral leishmaniasis in children. Mem Inst Oswaldo Cruz 2010, 105(suppl 3):310-313.

17. Pita-Pereira D, Alves CR, Souza MB, Brazil RP, Bertho AL, Barbosa AF, Britto C: Identification of naturally infected Lutzomyia intermedia and Lutzomyia migonei with Leishmania (Viannia) braziliensis in Rio de Janeiro (Brazil) revealed by a PCR multiplex non-isotopic hybridization assay. Trans $R$ Soc Trop Med Hyg 2005, 99:905-913.

18. Pita-Pereira D, Cardoso MAB, Alves CR, Brazil RP, Britto C: Detection of natural infection in Lutzomyia cruzi and Lutzomyia forattinii (Diptera: Psychodidae:Phlebotominae) by Leishmania infantum chagasi in an endemic area of visceral leishmaniasis in Brazil using a PCR multiplex assay. Acta Trop 2008, 107:66-69.

19. Passos VM, Lasmar EB, Gontijo CM, Fernandes O, Degrave W: Natural infection of a domestic cat (Felis domesticus) with Leishmania (Viannia) in the metropolitan region of Belo Horizonte, State of Minas Gerais, Brazil. Mem Inst Oswaldo Cruz 1996, 91:19-20.

20. Hall TA: BioEdit: a user-friendly biological sequence alignment editor and analysis program for Windows 95/98/NT. Nucl Acids Symp Ser 1999, 41:95-98.

21. Kumar S, Tamura K, Nei M: MEGA3: Integrated software for molecular evolutionary genetics analysis and sequence alignment. Brief Bioinform 2004, 5(suppl 2):150-163.

22. Cupolillo E, Grimaldi G Jr, Momen H, Beverley SM: Intergenic region typing (IRT): a rapid molecular approach to the characterization and evolution of Leishmania. Mol Biochem Parasitol 1995, 73:145-155.

23. Marfurt J, Niederwieser I, Makia ND, Beck HP, Felger I: Diagnostic genotyping of Old and New World Leishmania species by PCR-RFLP. Diagn Microbiol Infect Dis 2003, 46:115-124.

24. Rotureau B, Ravel C, Couppié P, Pratlong F, Nacher M, Dedet JP, Carme B: Use of PCR-restriction fragment length polymorphism analysis to identify the main New World Leishmania species and analyze their taxonomic properties and polymorphism by application of the assay to clinical samples. J Clin Microb 2006, 44(suppl 2):459-467.

25. Foulet F, Botterel F, Buffet P, Morizot G, Rivollet D, Deniau M, Pratlong F, Costa JM, Bretagne S: Detection and identification of Leishmania species from clinical specimens by using a real-time PCR assay and sequencing of the cytochrome b gene. J Clin Microb 2007, 45(suppl 7):2110-2115. 
26. Gangneux JP, Menotti J, Lorenzo F: Prospective value of PCR amplification and sequencing for diagnosis and typing of old world Leishmania infections in an area of nonendemicity. J Clin Microbiol 2003, 41:1419-1422.

27. Tupperwar N, Vineeth V, Rath S, Vaidya T: Development of a real-time polymerase chain reaction assay for the quantification of Leishmania species and the monitoring of systemic distribution of the pathogen. Diagn Microbiol Infect Dis 2008, 61(suppl 1):23-30.

28. Bensoussan $E$, Nasereddin A, Jonas F, Schnur LF, Jaffe CL: Comparison of PCR assays for diagnosis of cutaneous leishmaniasis. J Clin Microbiol 2006, 44:1435-1439.

29. Bulle B, Millon L, Bart JM, Gallego M, Gambarelli F, Portus M, Schnur L, Jaffe CL, Fernandez-Barredo S, Alunda JM, Piarroux R: Practical approach for typing strains of Leishmania infantum by microsatellite analysis. J Clin Microbiol 2002, 40:3391-3397.

doi:10.1186/1756-3305-5-15

Cite this article as: Pita-Pereira et al:: SYBR Green-based Real-Time PCR targeting kinetoplast DNA can be used to discriminate between the main etiologic agents of Brazilian cutaneous and visceral leishmaniases. Parasites \& Vectors 2012 5:15.

\section{Submit your next manuscript to BioMed Central} and take full advantage of:

- Convenient online submission

- Thorough peer review

- No space constraints or color figure charges

- Immediate publication on acceptance

- Inclusion in PubMed, CAS, Scopus and Google Scholar

- Research which is freely available for redistribution

Submit your manuscript at www.biomedcentral.com/submit 